\title{
256. Pseudo-differential Operators in the Theory of Hyperfunctions
}

\author{
By Masaki KASHIWARA*) and Takahiro KAWAI**) \\ (Comm. by Kunihiko KodAIRA, M. J. A., Dec. 12, 1970)
}

In this note we first give the definition of the sheaf of pseudodifferential operators, the notion of which is originally due to Sato. The sheaf is defined using Sato's theory of the sheaf $\mathcal{C}$. For the definition and the main properties of the sheaf $C$ we refer the reader to Sato [2].

Secondly we develop the theory of pseudo-differential operators of finite type and discuss some applications to the theory of linear partial differential operators.

This note is a summary of our forthcoming paper in which the details will be given.

1. Let $f$ be a real analytic mapping from an $n$-dimensional real analytic manifold $N$ to an $m$-dimensional real analytic manifold $M$. The mapping $f$ defines a natural homomorphism of vector bundles: $N \times T^{*} M \rightarrow T^{*} N$, where $T^{*} M$ and $T^{*} N$ denote the cotangent bundle of $M$ and that of $N$, respectively, and $N \times T_{M}^{*} M$ denotes the fibre product of $T^{*} M$ and $N$ over $M$. We denote its kernel by $T_{N}^{*} M$, which is not a vector bundle in general, and define $S_{N}^{*} M$ to be $\left(T_{N}^{*} M-N\right) / \boldsymbol{R}^{+} . \quad\left(\boldsymbol{R}^{+}\right.$ denotes the multiplicative group of positive numbers.) $S_{N}^{*} M$ is a closed subset of $N \underset{M}{\times} S^{*} M$, whose fibres over $N$ are spheres. The $f^{*}: N \underset{M}{N} T^{*} M$ $\rightarrow T^{*} N$ induces a projection $\rho: N \underset{M}{\times} S^{*} M-S_{N}^{*} M \rightarrow S^{*} N$. We denote by $\widetilde{\boldsymbol{\omega}}$ the natural projection $N \times S_{M}^{*} M-S_{N}^{*} M-S^{*} M$.

Let $\mathcal{A}_{M}$ be the sheaf of real analytic functions and let $v_{M}$ be the sheaf of densities with real analytic coefficients, which becomes an invertible $\mathcal{A}_{M}$-Module. By means of the theory of $\mathcal{C}$, we can define the following two fundamental homomorphisms, corresponding to the substitution and integration along fibre.

$$
\begin{aligned}
& f^{*}: \rho_{!} \widetilde{\omega}^{-1} \mathcal{C}_{M} \rightarrow \mathcal{C}_{N}, \\
& f_{*}: \widetilde{\omega}_{!} \rho^{-1}\left(C_{N} \otimes_{\mathcal{A}_{N}} v_{N}\right) \rightarrow \mathcal{C}_{M} \otimes_{\mathcal{A}_{M}} .
\end{aligned}
$$

Remark. If $f: X \rightarrow Y$ is a continuous map and $\mathscr{F}$ is a sheaf on $X, f_{!}(\mathscr{F})$ is a direct image with proper support, that is, $\Gamma\left(U, f_{!}(\mathcal{I})\right)$

*) Department of Mathematics, University of Tokyo.

**) Research Institute for Mathematical Sciences, Kyoto University. 
$=\left\{s \in \Gamma\left(f^{-1} U ; \mathcal{F}\right) ; \operatorname{supp}(s)\right.$ is proper over $\left.U\right\}$, for every open subset $U$ of $Y$.

We identify $N$ with the submanifold of $N \times M$ by the diagonal mapping. The projection $T^{*}(N \times M) \rightarrow N \times T^{*} M$ induces an isomorphism $S_{N}^{*}(N \times M) \rightarrow N \underset{M}{\times} S^{*} M$, by which we identify $S_{N}^{*}(N \times M)$ and $N \times S_{M}^{*} M$. In general, if $f: X \rightarrow Y$ is a fibre space with sphere as fibre, we denote by $a$ the antipodal mapping of $X$, which maps $x \in X$ to its antipodal point $x^{a}$. For any sheaf $\mathscr{F}$ over $X$, we denote by $\mathscr{F}^{a}$ the direct image of $\mathcal{F}$ by $a$.

Definition 1. We define the sheaf $\mathcal{Q}_{f}$ of pseudo-differential operators over $f$ by $\mathscr{Q}_{f}=\underline{\Gamma}_{S_{N}^{*}(N \times M)}\left(\mathcal{C}_{N \times M} \otimes_{\mathcal{A}_{M}}\right)^{a}$. Since the support of $\mathscr{P}_{f}$ is in $S_{N}^{*}(N \times M)$, we consider $\mathscr{P}_{f}$ as a sheaf on $N \underset{M}{\times} S^{*} M$.

If $M=N$ and $f$ is the identity map, $\mathscr{Q}_{f}$ is denoted by $\mathscr{Q}_{M}$ or by $\mathscr{Q}$ and a section of $\mathscr{P}_{M}$ is said to be pseudo-differential operator on $S^{*} M$. There are two fundamental bilinear homomorphisms

$$
\begin{aligned}
& \rho_{!}\left(\left.\mathcal{P}_{f}\right|_{N \times S^{*} M-S_{N}^{*}} \times \widetilde{\boldsymbol{\omega}}^{-1} \mathcal{C}_{M}\right) \rightarrow \mathcal{C}_{N} \\
& \widetilde{\boldsymbol{\omega}}_{!}\left(\rho^{-1} \mathcal{C}_{N} \otimes_{v_{N}} \times\left.\mathcal{Q}_{f}^{a}\right|_{N \times S^{*} M-S_{N}^{*}}\right) \rightarrow \mathcal{C}_{M} \otimes_{v_{M}} .
\end{aligned}
$$

Roughly speaking, the first bilinear homomorphism is defined by $(K(y, x) d x, \varphi(x)) \mapsto \int K(y, x) \varphi(x) d x$ and, the latter by $(\psi(y) d y, K(y, x) d x)$ $\mapsto\left[\int \psi(y) K(y, x) d x\right] d y$.

If $f: N \rightarrow M$ and $g: M \rightarrow L$ are two real analytic mappings, we can define the composition homomorphism of pseudo-differential operators over $g$ and pseudo-differential operators over $f$. Let $p_{1}: N \underset{L}{\times} S^{*} L-N \underset{M}{\times} S_{M}^{*} L$ $\rightarrow N \times S^{*} M$ and $p_{2}: N \times S^{*} L-N \times S_{M}^{*} L \rightarrow M \times S^{*} L$ be the projections deduced from $\widetilde{\omega}: M \times S^{*} L-S_{M}^{*} L \rightarrow S^{*} M$ and $f: N \rightarrow M$. Then the composition of pseudo-differential operators is described by the following bilinear homomorphism : $p_{1}^{-1} \mathcal{Q}_{f} \times p_{2}^{-1} \mathscr{Q}_{g} \rightarrow \mathscr{L}_{\text {gof }}$.

Now, consider the case where $N=M$ and $f$ is the identity. In this case $\mathscr{L}$ has a structure of sheaf of rings by the above composition homomorphism, which reduces to a sheaf bilinear homomorphism $\mathscr{P} \times \mathscr{P} \rightarrow \mathscr{P}$ since $S_{N}^{*} M$ is empty. By the operations $\mathscr{Q} \times \mathcal{C} \rightarrow \mathcal{C}$ and $\left(\mathcal{C} \otimes_{v}\right) \times \mathcal{L}^{a} \rightarrow \mathcal{C} \otimes_{v}, \mathcal{C}$ may be considered as a left $\mathscr{L}$-Module, and $\mathcal{C} \otimes_{v}$ as a right $\mathscr{Q}$-Module.

2. We next study a special class of pseudo-differential operators which is easy to manipulate. In the following we fix some local coordinate system and identify $M$ with a domain $\Omega$ in $R^{n}$ and $S^{*} M$ with $\Omega \times S^{n-1}$. We denote a point in $S^{*} M$ by $(x, \eta)$, where $\eta$ is a homogeneous coordinate of $S^{n-1}$. 
Definition 2 (Pseudo-differential operators of finite type). We call a pseudo-differential operator $P$ to be of finite type when its kernel function $K\left(x, x^{\prime}\right)$ is represented near $\left(x_{0}, \eta_{0}\right)$ as $\int \sum_{j \geq-m} p_{j}(x, \eta) \Phi_{j}\left(\left\langle x-x^{\prime}, \eta\right\rangle\right.$ $+i 0) \omega(\eta)$ where $p_{j}(x, \eta)$ and $\Phi_{j}(\tau)$ satisfy the following conditions (i) $\sim$ (iii), and $j$ runs over the integers. $\omega(\eta)$ is the volume element $\sum_{j=1}^{n}(-1)^{j-1} \eta_{j} d \eta_{1} \wedge \cdots \wedge d \eta_{j-1} \wedge d \eta_{j+1} \wedge \cdots \wedge d \eta_{n}$ :

$$
\Phi_{j}(\tau)=\left\{\begin{array}{l}
\frac{(-1)^{j}(n-j-1) !}{(-2 \pi \sqrt{-1})^{n}} \cdot \frac{1}{\tau^{n-j}} \quad(j<n) \\
\frac{-1}{(2 \pi \sqrt{-1})^{n}(j-n) !} \tau^{j-n} \log \tau \quad(j \geqq n)
\end{array}\right.
$$

(ii) $p_{j}(x, \eta)$ is real analytic near $\left(x_{0}, \eta_{0}\right)$ and positively homogeneous of degree $(-j)$ with respect to $\eta$, that is, $p_{j}(x, c \eta)=c^{-j} p_{j}(x, \eta)$ $(c>0)$.

(iii) There exist some complex neighbourhood $V$ of $\left(x_{0}, \eta_{0}\right)$ and some constant $c$ such that the following estimate $(*)$ holds ;

(*) $\sup _{(z, \zeta) \in V}\left|p_{j}(z, \zeta)\right| \leqq j ! c^{j}$ for sufficiently large $j$.

Definition 3. Let $P$ be a pseudo-differential operator of finite type whose kernel function has the above form. Then we define the order of $P$ by $(-1) \times \min \left\{j \mid p_{j}(x, \eta) \neq \equiv\right\}$. If $P$ is of order $m$, then we define the principal symbol of $P$ to be $p_{-m}(x, \eta)$.

Afterwards we sometimes write $P$ as $\sum_{j \geq-m} p_{j}\left(x, D_{x}\right)$.

Proposition 4. Let the kernel function of $P$ be given by

$$
\int \sum_{k \geq-m} a_{k}\left(x, x^{\prime}, \eta\right) \Phi_{k}\left(\left\langle x-x^{\prime}, \eta\right\rangle+i 0\right) \omega(\eta)
$$

where $a_{k}\left(x, x^{\prime}, \eta\right)$ is real analytic near $\left(x_{0}, x_{0}, \eta_{0}\right)$, is positively homogeneous of degree $(-k)$ with respect to $\eta$ and satisfies the estimate $\sup _{\left(z, z^{\prime}, 5\right) \in V}\left|a_{k}\left(z, z^{\prime}, \zeta\right)\right| \leqq k ! c^{k}(k \gg 0)$, for some constant $c$ and for some complex neighbourhood $V$ of $\left(x_{0}, x_{0}, \eta_{0}\right)$. Then the kernel function of $P$ has the form stated in Definition 2, that is $\int \sum p_{j}(x, \eta) \Phi_{j}\left(\left\langle x-x^{\prime}, \eta\right\rangle+i 0\right) \omega(\eta)$, where

$$
p_{j}(x, \eta)=\left.\sum_{j=k+|\alpha|} \frac{1}{\alpha !} D_{x}^{\alpha} D_{\eta}^{\alpha} a_{k}\left(x, x^{\prime}, \eta\right)\right|_{x^{\prime}=x} .
$$

This proposition is proved using the Taylor expansion of $a_{k}$ with respect to $\left(x-x^{\prime}\right)$ and Cauchy's integral formula.

Corollary 1. The property that a pseudo-differential operator $P$ is of finite type is independent of the choice of the local coordinate system.

Corollary 2. The principal symbol of $P$ is independent of the choice of the local coordinate system, as a homogeneous function on $S^{*} M$. 
Theorem 5. Let $P$ and $Q$ be pseudo-differential operators of finite type expressed in the form $\sum p_{j}\left(x, D_{x}\right)$ and $\sum q_{k}\left(x, D_{x}\right)$, respectively. Then the composed operator $R=P Q$ is also of finite type and if we write $R=\sum r_{l}\left(x, D_{x}\right)$, then

$$
r_{l}(x, \eta)=\sum_{l=j+k+|\alpha|} \frac{1}{\alpha !} D_{\eta}^{\alpha} p_{j}(x, \eta) D_{x}^{\alpha} q_{k}(x, \eta) .
$$

To prove this theorem we use the following formula which holds as an identity of sections of the sheaf $\mathcal{C}$;

$$
\begin{aligned}
& \int(\langle x, \xi\rangle+i 0)^{\lambda}(t-\langle x, \eta\rangle+i 0)^{\mu} d x \\
& =\frac{(-2 \pi i)^{n} \Gamma(-\lambda-\mu-n)}{\Gamma(-\lambda) \Gamma(-\mu)}(t+i 0)^{\lambda+\mu+n} \delta_{\lambda}(\xi: \eta),
\end{aligned}
$$

where $\delta_{\lambda}(\xi: \eta)$ is a $\delta$-function concentrated on $\xi=c \eta(c>0)$, homogeneous of degree $\lambda$ in $\xi$ and of degree $(-n-\lambda)$ in $\eta$.

Theorem 6. Let $P$ be a pseudo-differential operator of finite type and $p_{-m}(x, \eta)$ be its principal symbol. Then $\operatorname{supp}_{\operatorname{Ker}_{C} P} P$ and

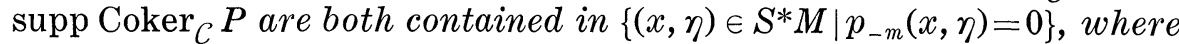
$\operatorname{Ker}_{\mathcal{C}} P$ is by definition the solution sheaf of the pseudo-differential operator $P$ which operates on the sheaf $\mathcal{C}$, and $\operatorname{Coker}_{\mathcal{C}} P$ denotes the cokernel of $P$.

This can be proved by the majorant method using the calculation of Theorem 5 .

Remark. This theorem was first found by Sato [1] for partial differential operators and is called Sato's fundamental theorem.

Theorem 7. Let $P$ and $Q$ be partial differential operators of order $m$ with simple characteristics and $P_{-m}(x, \eta)=Q_{-m}(x, \eta)$ is a polynomial of $(x, \eta)$ (for some local coordinate system). Then we can find locally some invertible pseudo-differential operators of finite type $R$ and $S$ such that $R Q=P S$.

Remark. The assumption that $P$ and $Q$ are partial differential operators and that $P_{-m}(x, \eta)=Q_{-m}(x, \eta)$ is a polynomial is very unsatisfactory. We hope that the assumption can be relaxed at least to the condition that $P$ and $Q$ are pseudo-differential operators of finite type of order $m$ with simple characteristics and $P_{-m}=Q_{-m}$. In fact, we can find $R$ and $S$ formally in that case but we have not yet proved the estimate $(*)$ in Definition 2.

Sketch of the proof of Theorem 7 .

We can assume that $Q(x, \eta)=Q_{-m}(x, \eta)$ without loss of generality. If $P_{-m}(x, \eta) \neq 0$ then Theorem 6 proves this theorem. So we can assume $\operatorname{grad}_{\eta} P_{-m} \neq 0$ by the assumption of simple characteristics. (In fact, it is sufficient to assume $\operatorname{grad}_{(\eta, x)} P_{-m}(x, \eta)=\left(\operatorname{grad}_{\eta} P_{-m}(x, \eta)\right.$, $\left.\operatorname{grad}_{x} P_{-m}(x, \eta)\right)$ is not parallel to $(0, \eta)$ if $P_{-m}(x, \eta)=0$ instead of the 
above assumption.) Assuming $R=S=\sum_{j \geqq 0} r_{j}\left(x, D_{x}\right)$ we obtain successively the first order partial differential equations of the following type:

$$
\begin{gathered}
\left\langle\operatorname{grad}_{\eta} P_{-m}(x, \eta), \operatorname{grad}_{x} r_{j}\right\rangle-\left\langle\operatorname{grad}_{\eta} r_{j}, \operatorname{grad}_{x} P_{-m}\right\rangle \\
+P_{-m+1}(x, \eta) r_{j}(x, \eta)=\rho_{j}(x, \eta),
\end{gathered}
$$

where $\rho_{0}=0$ and $\rho_{j}(x, \eta)(j \geqq 1)$ is determined by $\left\{r_{k}\right\}_{k=0, \cdots, j-1}$. By the assumption on $\operatorname{grad}_{\eta} P_{-m}$ we can find some non-characteristic surface $T=\{t(x, \eta)=0\}$ and give the initial condition of $r_{j}$ on $T$ by 1 for $j=0$ and by 0 for $j \geqq 1$. Using Euler's identity for homogeneous functions we can find $\left\{r_{j}(x, \eta)\right\}$ satisfying the conditions of Definition 2.

\section{References}

[1] M. Sato: Hyperfunctions and partial differential equations. Proc. Int. Conf. on Functional Analysis and Related Topics, Univ. of Tokyo Press, pp. 91-94 (1969).

[2] - : On the structure of hyperfunctions. Sûgaku no Ayumi, 15, 9-72 (1970) (Notes by M. Kashiwara, in Japanese). 